\title{
CLASSIFICATION OF RATIONAL UNICUSPIDAL PROJECTIVE CURVES WHOSE SINGULARITIES HAVE ONE PUISEUX PAIR
}

\author{
J. FERNÁNDEZ DE BOBADILLA, I. LUENGO, A. MELLE-HERNÁNDEZ, AND A. NÉMETHI
}

\section{INTRODUCTION}

It is a very old and interesting open problem to characterize those collections of embedded topological types of local plane curve singularities which may appear as singularities of a projective plane curve $C$ of degree $d$. (We invite the reader to consult the articles of Fenske, Flenner, Orevkov, Tono, Zaidenberg, Yoshihara, and the references therein, for recent developments.) The goal of the present article is to give a complete (topological) classification of those cases when $C$ is rational and it has a unique singularity which is locally irreducible (i.e. $C$ is unicuspidal) with one Puiseux pair.

In fact, as a second goal, we also wish to present some of the techniques which are $/ \mathrm{might}$ be helpful in such a classification, and we invite the reader to join us in our effort to produce a classification for all the cuspidal rational plane curves. In fact, this effort also motivates that decision, that in some cases (in order to have a better understanding of the present situation), we produce more different arguments for some of the steps.

In the next paragraph we formulate the main result. We will write $d$ for the degree of $C$ and $(a, b)$ for the Puiseux pair of its cusp, where $1<a<b$. We denote by $\left\{\varphi_{j}\right\}_{j \geq 0}$ the Fibonacci numbers $\varphi_{0}=0, \varphi_{1}=1, \varphi_{j+2}=\varphi_{j+1}+\varphi_{j}$.

1.1. Theorem. The Puiseux pair $(a, b)$ can be realized by a unicuspidal rational plane curve of degree $d$ if and only if $(d, a, b)$ appears in the following list.

(a) $(a, b)=(d-1, d)$;

(b) $(a, b)=(d / 2,2 d-1)$, where $d$ is even;

(c) $(a, b)=\left(\varphi_{j-2}^{2}, \varphi_{j}^{2}\right)$ and $d=\varphi_{j-1}^{2}+1=\varphi_{j-2} \varphi_{j}$, where $j$ is odd and $\geq 5$;

(d) $(a, b)=\left(\varphi_{j-2}, \varphi_{j+2}\right)$ and $d=\varphi_{j}$, where $j$ is odd and $\geq 5$;

(e) $(a, b)=\left(\varphi_{4}, \varphi_{8}+1\right)=(3,22)$ and $d=\varphi_{6}=8$;

(f) $(a, b)=\left(2 \varphi_{4}, 2 \varphi_{8}+1\right)=(6,43)$ and $d=2 \varphi_{6}=16$.

All these cases are realizable: (a) e.g. by $\left\{z y^{d-1}=x^{d}\right\}$, (b) by $\left\{\left(z y-x^{2}\right)^{d / 2}=x y^{d-1}\right\}$, or by the parametrization $[z(t): x(t): y(t)]=\left[1+t^{d-1}: t^{d / 2}: t^{d}\right]$. The existence of $(\mathrm{c})$ and (d) is guaranteed by Kashiwara classification [4, Corollary 11.4. These two cases can be realized by a rational pencil of type $(0,1)$ : the generic member of the pencil is (c), while the special member of the pencil is of type (d) (cf. also with the last paragraphs of the present article). Orevkov in [6 provides a different construction for curves which realize the case (d) (denoted by him by $C_{j}$ ). Similarly, the cases (e) and (f) are realized by the sporadic cases $C_{4}$ and $C_{4}^{*}$ of Orevkov [6].

Key words and phrases. Cuspidal rational plane curves, logarithmic Kodaira dimension.

The first author is supported by the Netherlands Organization for Scientific Research (NWO). The first three authors are partially supported by BFM2001-1488-C02-01. The forth author is partially supported by NSF grant DMS-0304759. 
1.2. Remarks. (1) Since $C$ is rational and its singular locus $p$ has Milnor number $\mu=$ $(a-1)(b-1)$, the genus formula says that

$$
(a-1)(b-1)=(d-1)(d-2) .
$$

On the other hand, not any triple $(d, a, b)$ with $(a-1)(b-1)=(d-1)(d-2)$ can be geometrically realized. E.g., $(5,3,7)$ or $(17,6,49)$ cannot.

(2) There are two integers which coordinate the above classification. The first one is defined as follows. Let $\pi: X \rightarrow \mathbb{P}^{2}$ be the minimal good embedded resolution of $C \subset \mathbb{P}^{2}$, and let $\bar{C}$ be the strict transform of $C$. Clearly, $\left(\pi^{*} C, \bar{C}\right)=C^{2}=d^{2}$, and $\pi^{*} C=\bar{C}+a b E_{-1}+\ldots$ (where $E_{-1}$ is the unique -1 exceptional curve of $\pi$ ), hence $d^{2}=\bar{C}^{2}+a b$. Using (1), we get:

$$
\left\{\begin{array}{l}
a+b=3 d-1-\bar{C}^{2} \\
a b=d^{2}-\bar{C}^{2}
\end{array}\right.
$$

Then $\bar{C}^{2}$ in the above cases is as follows: it is positive for (a) and (b), it is zero for (c), equals -1 for $(\mathrm{d})$, and $=-2$ for (e) and (f).

(3) The second guiding integer is the logarithmic Kodaira dimensions $\bar{\kappa}:=\bar{\kappa}\left(\mathbb{P}^{2} \backslash C\right)$ (cf. [3]). Its values are the following (cf. [6]): $-\infty$ for (a)-(d), and 2 for the last two sporadic cases. (In particular, $\bar{\kappa}$ depends only on the integers $(d, a, b)$, and it is independent on the analytic type of $C$ which realizes these integers.)

In particular, the above classification shows that $\bar{\kappa}=-\infty$ if and only if $\bar{C}^{2}>-2$.

In fact, after we finished the manuscript, we learned from the introduction of [8] that in 14. (written in Japanese) it is proved that for any unicuspidal rational curve $C, \bar{\kappa}=-\infty$ if and only if $\bar{C}^{2}>-2$. Using [14] (i.e. this equivalence), a possible 'quick' classification for $\bar{C}^{2}>-2$ would run as follows: Since for all these cases $\bar{\kappa}=-\infty$, we just have to separate in Kashivara's classification [4] those unicuspidal curves with exactly one Puiseux pair. Their numerical invariants $(d, a, b)$ are exactly those listed in (a)-(d).

On the other hand, this argument probably does not show what is really behind the classification of this case. Therefore, we decided to keep the structure of our manuscript, and provide an independent classification.

Note also that in [1] we list the complete topological classification of the cuspidal rational curves with $\bar{\kappa}<2$. In fact $\bar{\kappa}=0$ cannot occur because of a result of Tsunoda's [9], see also Orevkov's paper [6]. Moreover, Tono in [8] provides all the possible curves $C$ with $\bar{\kappa}=1$ : there is no one with one Puiseux pair.

Hence, in our case, the remaining part of the classification corresponds to $\bar{C}^{2} \leq-2$, or equivalently, to $\bar{\kappa}=2$. In general, the classification of this ('general') case is the most difficult; and in our case it is not clear at all at the beginning (and, in fact, it is rather surprising) that there are only two (sporadic) cases satisfying these data.

(4) Let $\alpha=(3+\sqrt{5}) / 2$ be the root of $\alpha+\frac{1}{\alpha}=3$. Notice that in family (d) $d / a$ and $b / d$ asymptotically equals $\alpha$. In fact, for $j$ odd, $\left\{\varphi_{j} / \varphi_{j-2}\right\}_{j}$ are the increasing convergents of the continued fraction of $\alpha$. Using this, another remarkable property of the family (d) can be described as follows (cf. 6], page 658). The convex hull of all the pairs $(m, d) \in \mathbb{Z}^{2}$ satisfying $m+1 \leq d<\alpha m$ (cf. with the sharp Orevkov inequality [6], or 2.7) coincides with the convex hull of all pairs $(m, d)$ realizable by rational unicuspidal curves $C$ (where $d=\operatorname{deg}(C)$ and $m=\operatorname{mult}(C, p))$ with $\bar{\kappa}\left(\mathbb{P}^{2} \backslash C\right)=-\infty$; moreover, this convex hull is generated by curves with numerical data (a) and (d).

(5) It is clear that the families (a)-(d) are organized in nice series of curves. It is less clear from the statement of the theorem, but rather clear from the proof, that also (e)-(f) form a 'series': they are the only curves with $3 d=8 a$ (cf. also with the next remark). 
(6) A hidden massage of the classification (and some of the steps of the proof) is that there is an intimate relationship between the semigroup of $\mathbb{N}$ generated by the elements $a$ and $b$, and the intervals of type $((l-1) d, l d]$. The endpoints $d$ and $3 d$ play crucial roles in some of the arguments. (E.g., $\bar{C}^{2} \leq-2$ if and only if $a+b>3 d$; see also 2.8) This fact is deeply exploited in [1. In fact, that paper strongly motivated the present manuscript.

(7) The (part of the) proof in section 4 clearly shows the deficiencies of the known restrictions, bounds which connect the local data $(a, b)$ with the degree $d$-although we list and try to use a large number of them. On the other hand, the above classification fits perfectly with the conjectured restriction proposed by the authors in [1] (valid in a more general situation), which, in fact, alone would provide the classification.

The authors thanks Maria Aparecida Ruas and Jean-Paul Brasselet for the wonderfull atmosphera in the "VIIIéme Rencontre Internationale de São Carlos sur les singularités reélles et complexes au CIRM". We also thank J. Stevens for pointing us out the full strength of the semicontinuity of the spectrum to eliminate some cases in the classification. Finally we thank David Lehavi who wrote for the fourth author some computer programs to help in the classification.

\section{RESTRICTIONS AND BOUNDS}

In the present section we list some general results which impose some restrictions for the integers $(d, a, b)$. We start with a trivial one: (1) and (2) clearly imply:

2.1. The 'trivial' bound. In any situation $b \geq d$. Moreover, if $b=d$ then $(a, b)=$ $(d-1, d)$.

If $b>d$ then $a<d-1$. The next result proves a 'gap' for $a$ : if $a<d-1$ then $a \leq d / 2$ too.

2.2. Lemma. The 'dual curve bound'. If $b>d$ then $d \geq 2 a$ (hence $b>2 a$ too).

Proof. Let $(C, p)$ be the germ of the singular point $p$ of $C$, and let $\left\{m_{i}\right\}_{i}$ be the multiplicity sequence of $(C, p)$. We will use the symbol $\vee$ for the corresponding invariants of the dual curve $C^{\vee}$ of $C$. By a result of C.T.C. Wall 13 Proposition 7.4.5, the blow ups of the singularities $(C, p)$ and $\left(C^{\vee}, p^{\vee}\right)$ (where $p^{\vee}$ corresponds to the tangent cone of $(C, p)$ ) are equisingular. Assume that $b<2 a$. Then $m_{2}=b-a$, hence $m_{2}^{\vee}=b-a \leq m_{1}^{\vee}$. But, according [13, the intersection multiplicity of the tangent cone of $(C, p)$ with $C$ at $p$ is $i=m_{1}+m_{1}^{\vee}$, hence $d \geq i=m_{1}+m_{1}^{\vee} \geq a+b-a=b$, a contradiction. In particular, $b \geq 2 a$. In this case $m_{2}=a$, hence $m_{2}^{\vee}=a$ as well. The above argument gives: $d \geq i \geq m_{1}+m_{1}^{\vee} \geq 2 a$.

2.3. The semicontinuity of the spectrum. The very existence of the curve $C$ shows that the local plane curve singularity $(C, p)$ is in the deformation of the local plane curve singularity $(U, 0):=\left(x^{d}+y^{d}, 0\right)$ (see e.g. [2] (3.24)). In particular, we can use the semicontinuity of the spectrum for this pair [11, 12. More precisely, this assures that in any interval $(c, c+1)$, the number of spectral numbers of $(C, p)$ is not larger than the number of spectral numbers of $(U, 0)$. E.g., for the intervals $(-1+l / d, l / d)(l=2,3, \ldots, d)$ one has the following inequality:

$$
\#\left\{\frac{i}{a}+\frac{j}{b}<\frac{l}{d} ; i \geq 1, j \geq 1\right\} \leq 1+2+\cdots+l-2=\frac{(l-2)(l-1)}{2} .
$$

Notice that the inequality $\left(S S_{d}\right)$ is automatically satisfied (with equality), since for both singularities the number of spectral numbers strict smaller than 1 is $(d-1)(d-2) / 2$. 
2.4. Example. The inequality $\left(S S_{d-1}\right)$. We denote by $\#_{d-1}$ the number of lattice points at the left hand side of $\left(S S_{d-1}\right)$. Since $i / a<(d-1) / d$ and $a<d$, one gets that $1 \leq i \leq a-1$. Therefore,

$$
\#_{d-1}=\sum_{i=1}^{a-1} \#\left\{j: 1 \leq j<b\left(\frac{d-1}{d}-\frac{i}{a}\right)\right\}=\sum_{i=1}^{a-1}\left\lceil b-\frac{b}{d}-\frac{i b}{a}\right\rceil-1,
$$

hence

$$
\#_{d-1}=(a-1)(b-1)-\sum_{i=1}^{a-1}\left\lfloor\frac{b}{d}+\frac{i b}{a}\right\rfloor .
$$

This expression can be computed explicitly. Indeed, since $(a, b)$ is a lattice point and $\operatorname{gcd}(a, b)=1$, one has:

$$
\sum_{i=1}^{a}\left\lfloor\frac{i b}{a}\right\rfloor=\frac{(a+1)(b+1)}{2}-a
$$

hence

$$
\sum_{i=1}^{a}\left\lfloor\frac{i b}{a}+\frac{b}{d}\right\rfloor=\frac{(a+1)(b+1)}{2}-a+a\left\lfloor\frac{b}{d}\right\rfloor+\sum_{i=1}^{a}\left\lfloor\left\{\frac{i b}{a}\right\}+\left\{\frac{b}{d}\right\}\right\rfloor .
$$

Notice that the set $\{i b / a\}$ for $i=1, \ldots, a$ is the same as the set $r / a$ for $r=0, \ldots, a-1$. Moreover, $r / a+\{b / d\} \geq 1$ if and only if $a-1 \geq r \geq\lceil a(1-\{b / d\})\rceil$, hence the number of possible $r$ 's is $\lfloor a\{b / d\}\rfloor$. Therefore,

$$
\sum_{i=1}^{a}\left\lfloor\frac{i b}{a}+\frac{b}{d}\right\rfloor=\frac{(a+1)(b+1)}{2}-a+\left\lfloor\frac{a b}{d}\right\rfloor .
$$

Hence

$$
\sum_{i=1}^{a-1}\left\lfloor\frac{i b}{a}+\frac{b}{d}\right\rfloor=\frac{(a+1)(b+1)}{2}-a-b-\left\lfloor\frac{b}{d}\right\rfloor+\left\lfloor\frac{a b}{d}\right\rfloor
$$

or

$$
\#_{d-1}=\frac{(a-1)(b-1)}{2}+\left\lfloor\frac{b}{d}\right\rfloor-\left\lfloor\frac{a b}{d}\right\rfloor
$$

Then, using (1) and (2), $\left(S S_{d-1}\right)$ becomes:

$$
\left\lfloor\frac{b}{d}\right\rfloor+\left\lceil\frac{\bar{C}^{2}}{d}\right\rceil \leq 2
$$

2.5. Other examples of $\left(S S_{l}\right)$. $\left(S S_{2}\right)$ is equivalent with $1 / a+1 / b \geq 2 / d$. This is true automatically, since $1 / a+1 / b \geq 1 / d+1 /(d-1)>2 / d$. The next inequality $\left(S S_{3}\right)$ is equivalent with $2 / b+1 / a \geq 3 / d$, which also is satisfied automatically.

If $b>d$ then $a+2 b>3 a+b$ (cf. 2.2), hence $\left(S S_{4}\right)$ is equivalent with the pair of inequalities: $a+2 b \geq 4 a b / d$ and $4 a+b \geq 4 a b / d$. Or, via (2):

$$
\min \{3 a, b\} \geq d+1+\frac{d-4}{d} \bar{C}^{2} .
$$

This with an (absolute) lower bound for $\bar{C}^{2}$ already is interesting: $3 a>d+$ const, which has the flavour of the Matsuoka-Sakai inequality $3 a>d$ (see 2.6) proved by different methods.

By a similar method as above, one can verify that $\left(S S_{d-2}\right)$ is equivalent with:

$$
\left\lfloor\frac{2 b}{d}\right\rfloor+\left\lceil\frac{2 \bar{C}^{2}}{d}\right\rceil \leq 5
$$


and $\left(S S_{d-3}\right)$ is equivalent with:

$$
\left\lfloor\frac{3 b}{d}\right\rfloor+\left\lfloor\frac{3 b}{d}-\frac{b}{a}\right\rfloor+\left\lceil\frac{3 \bar{C}^{2}}{d}\right\rceil \leq 8
$$

In general, one expects that the set of all inequalities $\left(S S_{l}\right)$ is really strong.

The next set of restrictions are provided by Bogomolov-Miyaoka-Yau type inequalitities:

2.6. Matsuoka-Sakai inequality. The inequality [7] in our case reads as $d<3 a$ (valid for any $\bar{\kappa}$ ).

2.7. Remark. Orevkov's inequality. Orevkov in [6] obtained different improved versions of 2.6. Below $\alpha=(3+\sqrt{5}) / 2 \approx 2.618$ and $\beta=1 / \sqrt{5}$.

(a) 6] Theorem $\mathrm{B}(\mathrm{a})$ : If $\bar{\kappa}=-\infty$, then $d<\alpha a$.

(b) [6] Theorem $\mathrm{B}(\mathrm{b})$ : If $\bar{\kappa}=2$, then $d<\alpha(a+1)-\beta$.

(c) [6] $(2.2)(4):$ If $\bar{\kappa}=2$, then

$$
-\bar{C}^{2} \leq-2+\frac{a}{b}+\frac{b}{a}
$$

Finally, we end with the following:

2.8. The 'semigroup density property'. [1] Let $\Gamma$ be the semigroup of $(C, p)$, i.e. the semigroup (with 0 ) of $\mathbb{N}$ generated by the integers $a$ and $b$. Then for any $0 \leq l<d$ the following inequality holds:

$$
\# \Gamma \cap[0, l d] \geq(l+1)(l+2) / 2 .
$$

Proof. It is instructive to sketch the proof for $l=3$ case: we wish to prove $\# \Gamma \cap[0,3 d] \geq 10$. Recall that a cubic is determined by 9 parameters. Therefore, $\# \Gamma \cap[0,3 d] \leq 9$ would imply the existence of a cubic with intersection multiplicity with $C$ at $p$ strict greater than $3 d$, which contradicts Bézout's theorem.

In the classical theory, many 'candidates' $(d, a, b)$ were eliminated by different geometric constrictions using ingenious Cremona transformations. We will exemplify this in 4.8 .

\section{The Classification in the CASE $\bar{C}^{2}>0$}

3.1. Theorem. If $\bar{C}^{2}>0$ then either $(a, b)=(d-1, d)$ or $(a, b)=(d / 2,2 d-1)$.

Proof. Since $b \geq d$ (cf. 2.1), by (3) we get that $\bar{C}^{2} \leq d$. Clearly, equality holds if and only if $(a, b)=(d-1, d)$. Next, assume that $0<\bar{C}^{2}<d$. Then again by $(3)$ one has $\lfloor b / d\rfloor \leq 1$, or $b<2 d$. But notice that $b<2 d-1$ would imply (by (1)) that $a>d / 2$ which contradicts 2.2. Hence $b=2 d-1$.

\section{Classification in the Case $\bar{C}^{2} \leq-2$}

4.1. Our first goal is to prove that $3 d \geq 8 a$. For this we apply 2.8 for $l=3$. Since $a+b>3 d$ (cf. (2)) and $9 a>3 d$ (cf. [2.6) ), the needed 10 elements of $\Gamma \cap[0,3 d]$ must be $b, 0, a, \ldots, 8 a$, hence $8 a \leq 3 d$. 


\subsection{Corollary. $\bar{\kappa}\left(\mathbb{P}^{2} \backslash C\right)=2$.}

Proof. $\bar{\kappa}$ cannot be $-\infty$ because of 2.7(a); cannot be 0 because of [6], Theorem B(c) (see also [9]). Unicuspidal rational curves with $\bar{\kappa}=1$ are classified by K. Tono [8], the corresponding splice diagrams are listed in [1]: there is no example with one Puiseux pair.

Now, the classification for $\bar{C}^{2} \leq-2$ can be finished in two different ways.

4.3. First proof. Using the computer. The first version is based on the inequality 2.7(b). Notice that in the case of a geometric realization one must have

$$
3 \alpha(a+1)-3 \beta>3 d \geq 8 a,
$$

which is true only if $a \leq 44$, (or, by using again $d<\alpha(a+1)-\beta$ ), only if $d \leq 117$. Hence, we have only to analyze the finite family determined by, say, $d \leq 117$. Then, one can search with the computer for 3-uples $(d, a, b)$ verifying all the restrictions considered above. E.g., we used the conditions $d \leq 117, \operatorname{gcd}(a, b)=1, a<d<b, d<3 a, 3 d \geq 8 a$, $\left.2 \leq-\bar{C}^{2} \leq-2+\frac{a}{b}+\frac{b}{a}, b<\alpha(d-1)(d-2) /(d-2 \alpha+\beta)\right)+1,(d-\alpha+\beta) / \alpha<a$, and $\left(S S_{d-1}\right),\left(S S_{d-2}\right),\left(S S_{d-3}\right),\left(S S_{d-4}\right),\left(S S_{4}\right)$. Using the inequality $3 d \geq 8 a$ and a similar computation as in the case of $\left(S S_{d-1}\right)$, we obtain that $\left(S S_{d-4}\right)$ is equivalent with

$$
\left\lfloor\frac{4 b}{d}\right\rfloor+\left\lfloor\frac{4 b}{d}-\frac{b}{a}\right\rfloor+\left\lceil\frac{4 \bar{C}^{2}}{d}\right\rceil \leq 13
$$

Then the only triplets satisfying all these are listed below (in the list appears $\left(d, a, b ; \bar{C}^{2}\right)$ :

$$
\begin{aligned}
& C_{1}:=(8,3,22 ;-2), \\
& C_{2}:=(11,4,31 ;-3), \\
& C_{3}:=(16,6,43 ;-2), \\
& C_{4}:=(17,6,49 ;-5), \\
& C_{5}:=(19,7,52 ;-3), \\
& C_{6}:=(20,7,58 ;-6) .
\end{aligned}
$$

Next, notice that the curves $C_{1}$ and $C_{3}$ exist, they are listed in our classification theorem. The others do not exist: $C_{2}$ is eliminated by Orevkov in [6], page 2 (see also 4.9 (b)); $C_{4}$ and $C_{6}$ can be excluded by the semicontinuity property of the spectrum (applied for all the intervals of type $(l / d, l / d+1),-d<l<d)$, finally $C_{5}$ can be eliminated by the "nodal cubic trick', see 4.9 (a). (Notice also that $C_{2}$ and $C_{5}$ cannot be eliminated by the semicontinuity property.)

4.4. Second proof. Resolving diophantic equations. Next we show how one can analyse the case $3 d \geq 8 a$ (cf. 4.1) by a diophantic equation (for the convenience of the reader, later we will make more precise the geometry behind this equation, cf. 4.7 and 4.8). Our goal is to eliminate everything excepting $C_{1}$ and $C_{3}$, and to emphasize that $C$ exists if and only if $3 d=8 a$, and $C_{1}$ and $C_{3}$ are the only solutions with $3 d=8 a$.

Let us write $x:=3 d-8 a \geq 0$. Then clearly $3 \mid a-x$. Moreover,

$$
\begin{gathered}
-\bar{C}^{2}(a-1)=-(3 d-1-a-b)(a-1)=-(x+7 a-1-b)(a-1) \\
=(b-1)(a-1)-(x+7 a-2)(a-1)=(d-1)(d-2)-(x+7 a-2)(a-1) .
\end{gathered}
$$

Using again $d=(x+8 a) / 3$ one gets

$$
-9 \bar{C}^{2}(a-1)=x^{2}+7 a x+a^{2}+9 a .
$$

4.5. The case $x=0$. (6) implies the divisibility $a-1 \mid 10$. Since one also has $3 \mid a$, the only solutions are $a=3$ and $a=6$, corresponding to $C_{1}$ and $C_{3}$ above. 
4.6. Facts. $-\bar{C}^{2} \leq 7$ and $x \leq 5$.

Proof. First we verify $-\bar{C}^{2} \leq 7$. It is easy to verify (using (1), (2) and $d / 3<a \leq d / 2$, cf. 2.6] and 2.2] that for $6 \leq d \leq 10$ this is true. Hence assume that $d \geq 11$. Notice that if for some (positive) $k$ one has $k d \leq-\bar{C}^{2}<(k+1) d$, then (3) gives $b / d \leq 3+k$. But $d / a<3$ by [2.6. hence $b / a<3(3+k)$. Using [2.7](c) one gets $-\bar{C}^{2} \leq 3 k+7$. Since for $k>0$ and $d \geq 11$ one has $3 k+7<d k$, one should have $k=0$.

Using this and $x \geq 6$, from $(6)$ we get $63(a-1) \geq 36+42 a+a^{2}+9 a$, which has no solution.

Now, we consider the above equation (6) for $x \geq 1$. By 4.6 we only have to analyse the cases $1 \leq x \leq 5$, and eliminate all the solutions.

The case $x=1$. In this case one has $-9\left(\bar{C}^{2}+2\right)(a-1)=(a-1)^{2}+18$, hence $3|a-1| 18$ but $9 \not \backslash a-1$. In particular, $a=4$ or 7 corresponding to $C_{2}$ and $C_{5}$ above.

The case $x=2$. Similarly as above, $a-1 \mid 28$ and $3 \mid a-2$, hence $a-1=4,7$ or 28. In fact, if $a=5$ then $d=12$ and $b \notin \mathbb{Z}$. The next case $\left(d, a, b ; \bar{C}^{2}\right)=(22,8,61 ;-4)$ can be eliminated by (5); the last $(78,29,210 ;-6)$ by 2.7(c).

The case $x=3$. Now $a-1 \mid 40$ and $3 \mid a$. The possible $a$ 's are $a=3$ which gives $d=$ 9 contradicting [2.6] $a=6$ providing $C_{4} ; a=9$ providing $(25,9,70 ;-5)$ which can be eliminated by (5), and $a=21$ providing $(57,21,155 ;-6)$ which is eliminated by 2.7 (c).

The case $x=4$. (6) has two solutions: $C_{6}:(20,7,58 ;-6)$ and $(28,10,79 ;-6)$, the second one can be eliminated by (5).

The case $x=5$ provides two integral solutions: $(23,8,67 ;-7)$ and $(31,11,88 ;-7)$. Both can be eliminated by 2.7(c).

We end this section by the description of the promised geometric construction (used also in [6] and by E. Artal-Bartolo as well).

4.7. Lemma. The existence of a specific nodal cubic. There exists an (unique) irreducible cubic $N \subset \mathbb{P}^{2}$ with a node singularity at $p$ such that $N$ and $C$ share the first seven infinitely near points at $p$.

Proof. A cubic is determined by nine parameters. The multiplicity sequence of $N$ at $p$ should be $\left[2,1_{6}\right]$. Passing through $p$ and having multiplicity 2 provides 3 conditions. The remaining six conditions are imposed by the remaining six infinitely near points. The condition which would imply that the singularity $(N, p)$ is a cusp would involve another equation (the vanishing of the determinant of the quadratic part at $p$ ), and the corresponding system of equations would not have any solution. Similar arguments eliminates other type of singularities (two smooth branches with contact two, or $(N, p)$ with multiplicity 3 ). Hence $(N, p)$ is a node.

Next we prove that $N$ cannot be a product of three linear forms. Indeed, the tangent line $L_{0}$ of $C$ at $p$ goes just through the first two infinitely near points because $d<3 a$ and $d=L_{0} \cdot C$. Any other line has less tangency than $L_{0}$. This also shows that $N$ cannot be $L_{0} \cdot Q$ for some $Q$ (transversal to $L_{0}$ at $\left.p\right)$.

The remaining posibility is $N=L Q$ where $Q$ is a smooth conic and $L$ and $Q$ meets transversally at $p$. Since $Q$ is determined by five conditions (five infinitely near points) then $Q$ and $C$ must be tangent and share the seven infinitely near points at $p$. In particular by Bezout $2 d=Q \cdot C \geq 6 a$ which is in contradiction with $d<3 a$, cf. [2.6] 
4.8. The Cremona transformation associated with the nodal cubic $N$. Consider the nodal cubic $N$ given by 4.7. First we verify that $C$ and $N$ share exactly the first seven infinitely near points. Indeed, assume that this is not the case. If $b \leq 8 a$ then the multiplicity sequence of $(C, P)$ is $\left[a_{7}, b-7 a, \ldots\right]$, hence $3 d \geq 2 a+6 a+b-7 a=a+b=3 d-1-\bar{C}^{2}>3 d$, a contradiction. If $b>8 a$ then the multiplicity sequence of $(C, P)$ is $\left[a_{8}, \ldots\right]$, hence $3 d \geq 9 a$ which contradicts 2.6 .

In particular, the intersection multiplicity of $C$ and $N$ at $P$ is $8 a$. Assume that $C \cap N=$ $\left\{P, P_{1}, \ldots, P_{r}\right\}$. Notice that at $P_{i}(1 \leq i \leq r)$ both curves $C$ and $N$ are smooth, let $k_{i}$ be their intersection multiplicity at $P_{i}$. By Bezout's theorem one has $3 d=8 a+\sum_{i} k_{i}$. We prefer to write $x:=\sum_{i} k_{i}$, hence $3 d=8 a+x$ (and the notation is compatible with above).

Blow up the common seven infinitely near points. We get seven irreducible exceptional divisors $\left\{E_{i}\right\}_{i=1}^{7}$. Let $\tilde{C}$ and $\tilde{N}$ be the strict transforms of $C$ and $N$. One has the following intersections: $E_{1}^{2}=\cdots=E_{6}^{2}=-2, \quad E_{7}^{2}=-1, \quad \tilde{N}^{2}=-1, \quad E_{1} \cdot E_{2}=E_{2} \cdot E_{3}=\cdots=$ $E_{6} \cdot E_{7}=1, E_{1} \cdot \tilde{N}=E_{7} \cdot \tilde{N}=1$. Also, $\tilde{C}$ intersects $E_{7}$ (but not the other irreducible exceptional divisors) at a point $P^{\prime}$, and the singularity $\left(\tilde{C}, P^{\prime}\right)$ has exactly one Puiseux pairs of type $(b-7 a, a)$. The intersection of $\tilde{N}$ with $E_{7}$ is not $P^{\prime}$.

Consider now the curve $\tilde{N} \cup \cup_{i=1}^{6} E_{i}$. Clearly, this can be blown down, and after this modification $\pi$ we get another copy of $\mathbb{P}^{2}$. Let the image of $\tilde{C}$ via this projection be $C^{\prime}$. By standard (intersection) argument one gets that the degree $d^{\prime}$ of $C^{\prime}$ is

$$
\left.d^{\prime}=8 d-21 a \quad \text { (which also satisfies } 3 d^{\prime}=8 x+a\right) \text {. }
$$

The curve $C^{\prime}$ has at most two singular points. One candidate is the (isomorphic) image of the germ at $P^{\prime}$ with one Puiseux pair $(b-7 a, a)$. The other is the common image of the points $\left\{P_{i}\right\}(1 \leq i \leq r)$. Clearly, if $x=0$ then this point does not exist, if $x=1$ then this is a smooth point, but otherwise it is singular. One can find its embedded resolution graph by blowing up (for each $i$ ) $k_{i}$ times the point $P_{i}$. Hence, by A'Campo's formula one can determine its Milnor number, which is $\mu=7 x^{2}-7 x-r+1$ (provided that $x \geq 1$ ). Since it has $r$ local irreducible components, the delta-invariant is $\left(7 x^{2}-7 x\right) / 2$. Then one can verify that (6) corresponds to the genus formula of $C^{\prime}$.

4.9. Example. (a) Let us start with $(d, a, b)=(19,7,52)$. Then $x=1$, hence $C^{\prime}$ is again rational and unicuspidal with $\left(d^{\prime}, a^{\prime}, b^{\prime}\right)=(5,3,7)$. But such a curve does not exist because of 3.1 (one can also check the classification of rational curves of degree five e.g. in [5]).

(b) Let us consider now the curve $C_{2}$ above with data $(d, a, b)=(11,4,31)$. Then $x=1$, hence $C^{\prime}$ is rational unicuspidal, say at $Q_{1}$, with $\left(d^{\prime}, a^{\prime}, b^{\prime}\right)=(4,3,4)$. Notice that a curve with this triplet may exists - although $C_{2}$ does not. The image $\bar{N}$ under the modification $\pi$ of the exceptional curve $E_{7}$ is a (rational) nodal cubic with a node, say at $Q_{2}\left(\neq Q_{1}\right)$. Moreover, $\bar{N} \cdot C^{\prime}=4 Q_{1}+8 Q_{2}$. At $Q_{1}, \bar{N}$ is non-singular and with the same tangent as $C^{\prime}$, and at $Q_{2}$ the quartic $C^{\prime}$ has intersection multiplicity 7 with one of the branches of the node of $\bar{N}$ and 1 with the other. To show that $C_{2}$ does not exist we will prove that such configuration of the rational curves $C^{\prime}$ and $\bar{N}$ in $\mathbb{P}^{2}$ does not exist.

Choosing affine coordinates we may assume that $C^{\prime}$ is given by the zero locus of $a y^{3}+$ $a_{1} y^{3} x+a_{2} y^{2} x^{2}+a_{3} y x^{3}+x^{4}+a_{0} y^{4}$; with $a \neq 0$. In such a case $Q_{1}=(0,0)$ and its tangent line $L_{1}=\{y=0\}$ verifies $L_{1} \cdot C^{\prime}=4 Q_{1}$. The curve $C^{\prime}$ has a parametrization given by $[z(\lambda, t): x(\lambda, t): y(\lambda, t)]=\left[\lambda^{4}+a_{3} t \lambda^{3}+a_{2} t^{2} \lambda^{2}+a_{1} t^{3} \lambda+a_{0} t^{4}:-a t^{3} \lambda:-a t^{4}\right]$.

To have $I_{Q_{1}}\left(\bar{N}, C^{\prime}\right)=4$ then $\bar{N}$ must be the zero locus of a polyomial $y+f_{2}(x, y)+f_{3}(x, y)$ (see the parametrization of $C^{\prime}$ ), where $f_{2}(x, y)=m_{1,1} x y+m_{2,0} x^{2}+m_{0,2} y^{2}$ and $f_{3}(x, y)=$ $n_{1,2} x y^{2}+n_{2,1} x^{2} y+n_{3,0} x^{3}+n_{0,3} y^{3}$. 
Next one imposes that, in the affine plane $\mathbb{P}^{2} \backslash L_{1}=\{y \neq 0\}$, the curves $C^{\prime}$ and $\bar{N}$ must meet at only one point $Q_{2}$ (with intersection multiplicity 8 ). The parametrization of $C^{\prime}$ in this affine chart is $(z, x)=\left(s^{4}+a_{3} s^{3}+a_{2} s^{2}+a_{1} s+a_{0},-a s\right)$ and the equation of $\bar{N}$ is given by $z^{2}+f_{2}(x, 1) z+f_{3}(x, 1)=0$. Imposing to have a solution of the form $(A s+B)^{8}$ gives $B=a_{3} A / 4$ which means $s=-a_{3} / 4$. We have two possibilities: firstly, if $a_{3}=0$ then $s=0$ and $Q_{2}=(z, x)=\left(a_{0}, 0\right)$. The solutions are given by $m_{1,1}=2 a_{1} / a ; m_{2,0}=$ $-2 a_{2} / a^{2} ; m_{0,2}=-\left(2 a_{0}-a_{2}^{2}\right) ; n_{1,2}=\left(-2 a_{0}+a_{2}^{2}\right) a_{1} / a ; n_{2,1}=\left(a_{1}^{2}+2 a_{0} a_{2}-a_{2}^{3}\right) / a^{2} ; n_{3,0}=$ $-2 a_{1} a_{2} / a^{3} ; n_{0,3}=\left(a_{0}-a_{2}^{2}\right) a_{0}$. To have $\bar{N}$ a node at $Q_{2}$ implies $a_{2}$ vanishes and therefore $\bar{N}$ must be a conic which is a contradiction.

In the other case, i.e. $a_{3} \neq 0$ then $s=-a_{3} / 4$ and $Q_{2}=(z, x)=\left(z_{0}, a a_{3} / 4\right)$. The solutions are given by:

$$
\begin{aligned}
& m_{1,1}=\left(16 a_{1}-a_{3}^{3}\right) /(8 a) ; \quad m_{2,0}=\left(3 / 4 a_{3}^{2}-2 a_{2}\right) / a^{2} ; \\
& m_{0,2}=-2 a_{0}+a_{2}^{2}+19 a_{3}^{4} / 128-3 a_{2} a_{3}^{2} / 4 ; \\
& n_{1,2}=-\left(4096 a_{0} a_{1}-2048 a_{1} a_{2}^{2}-304 a_{1} a_{3}^{4}+1536 a_{1} a_{2} a_{3}^{2}-256 a_{0} a_{3}^{3}+a_{3}^{7}\right) /(2048 a) ; \\
& n_{2,1}=\left(2^{11} a_{0} a_{2}+\left(2^{5} a_{1}\right)^{2}-2^{10} a_{2}^{3}-152 a_{2} a_{3}^{4}+768 a_{2}^{2} a_{3}^{2}-128 a_{1} a_{3}^{3}-768 a_{0} a_{3}^{2}+7 a_{3}^{6}\right) /\left(2^{5} a\right)^{2} ; \\
& n_{3,0}=\left(-64 a_{1} a_{2}+32 a_{3} a_{2}^{2}+3 a_{3}^{5}-20 a_{2} a_{3}^{3}+24 a_{1} a_{3}^{2}\right) /\left(32 a^{3}\right) ; \\
& n_{0,3}=a_{0}^{2}-a_{0} a_{2}^{2}-(19 / 128) a_{0} a_{3}^{4}+(3 / 4) a_{0} a_{2} a_{3}^{2}+(1 / 65536) a_{3}^{8} .
\end{aligned}
$$

In order $\bar{N}$ to have multiplicity two at $Q_{2}$ one needs $a_{2}=3 a_{3}^{2} / 8$ but this condition also impose that the tangent cone of $\bar{N}$ at $Q_{2}$ is a double line and therefore $Q_{2}$ cannot be a node. Hence this configuration also does not exist.

\section{The CASE $\bar{C}^{2}=0,-1$}

In this section we find all the integer solution $(d, a, b)$ of $(2)$ with $\bar{C}^{2}=0,-1$ and we show that all of them can be realized by some unicuspidal rational plane curve of degree $d$ and Puiseux pair $(a, b)$. Let $\varphi_{j}$ be the $i$-th Fibonacci number, that is $\varphi_{0}=0, \varphi_{1}=1$ and $\varphi_{j+2}:=\varphi_{j+1}+\varphi_{j}$. They share many interesting properties, see e.g. 10. We will use here the following :

$$
3 \varphi_{j}=\varphi_{j-2}+\varphi_{j+2}, \quad \text { and } \quad \varphi_{j}^{2}=(-1)^{j+1}+\varphi_{j-1} \varphi_{j+1} .
$$

Let $\Phi=\frac{1+\sqrt{5}}{2}$ be the positive solution of the equation $\Phi^{2}-\Phi-1=0$. For every integer $j>0$ one has :

$$
\Phi^{j}=\frac{\varphi_{j+1}+\varphi_{j-1}+\varphi_{j} \sqrt{5}}{2}
$$

5.1. The Pell equation. The system of equations (2) for $\bar{C}^{2}=0,-1$ can be transformed (see below) into the Pell equation:

$$
x^{2}-5 y^{2}=-4, x, y \in \mathbb{Z} .
$$

Consider the number field $K=\mathbb{Q}[\sqrt{5}]$ and its ring of integers $R=\mathbb{Z}[\sqrt{5}]$, which is a UFD. If $\gamma=x+y \sqrt{5}$ is a solution of (9) then its norm is $N_{K}(\gamma)=\gamma \bar{\gamma}=-4$. Consider $\eta=1+\sqrt{5}$, then $N_{K}(\eta)=-4$ and -4 has a prime decomposition $-4=\eta \bar{\eta}$. Since the fundamental unit of $K$ turns out to be $u=2+\sqrt{5}$ and $\gamma$ is associated either to $\eta$ or $\bar{\eta}$ then $\gamma$ is either $\pm u^{r} \eta$ 
or $\pm \bar{u}^{r} \bar{\eta}$ (since $\bar{u}=-1 / u$ ) for $r \in \mathbb{Z}$. Moreover $N_{K}(u)=-1$ which implies that $r$ must be even, that is $r=2 j$ for $j \in \mathbb{Z}$. Then $\eta=2 \Phi$ and from the identity $\Phi^{2}=\Phi+1$ one gets $\Phi^{3}=u$.

Thus solutions of (9) are either $\gamma= \pm u^{2 j} \eta= \pm 2 \Phi^{6 j+1}$ or $\gamma= \pm \bar{u}^{2 j} \bar{\eta}= \pm 2 \bar{\Phi}^{6 j+1}$ with $j \in \mathbb{Z}$. Using $\Phi \bar{\Phi}=-1, \gamma$ is either $\pm 2 \Phi^{6 j+1}, \pm 2 \Phi^{6 j-1}$, or their conjugates $\pm 2 \bar{\Phi}^{6 j+1}, \pm 2 \bar{\Phi}^{6 j-1}$ with $j \geq 0$. Using (7) and (8) the set of solutions of (9) is given by
(A) $\pm\left(\varphi_{6 j+2}+\varphi_{6 j}+\varphi_{6 j+1} \sqrt{5}\right)$, with $j \geq 0$,
(B) $\pm\left(\varphi_{6 j}+\varphi_{6 j-2}+\varphi_{6 j-1} \sqrt{5}\right)$, with $j \geq 0$,
(C) $\pm\left(\varphi_{6 j+2}+\varphi_{6 j}-\varphi_{6 j+1} \sqrt{5}\right)$, with $j \geq 0$,
(D) $\pm\left(\varphi_{6 j}+\varphi_{6 j-2}-\varphi_{6 j-1} \sqrt{5}\right)$, with $j \geq 0$.

5.2. The case $\bar{C}^{2}=0$. Since $\operatorname{gcd}(a, b)=1$ and $a b=d^{2}$ then $a=m^{2}, b=n^{2}$ and $d=m n$ for some positive integers $m, n$ with $\operatorname{gcd}(m, n)=1$. Thus $a+b=3 d-1$ transforms into

$$
m^{2}+n^{2}=3 m n-1 \text {. }
$$

5.3. The case $\bar{C}^{2}=-1$. The system (2) provides the equation

$$
a^{2}+d^{2}=3 a d-1 \text {. }
$$

Thus, any solution $(\omega, v)$ of $\omega^{2}+v^{2}=3 \omega v-1$ is a solution of $(2 \omega-3 v)^{2}-5 v^{2}=-4$. Hence, with the transformation $x=2 \omega-3 v, y=v$, one gets the solutions of (9).

Case A. If $\gamma= \pm\left(\varphi_{6 j+2}+\varphi_{6 j}+\varphi_{6 j+1} \sqrt{5}\right), j \geq 0$, is a solution of (9) then $v= \pm \varphi_{6 j+1}$ and $\omega= \pm\left(\varphi_{6 j+2}+\varphi_{6 j}+3 \varphi_{6 j+1}\right) / 2= \pm \varphi_{6 j+3}$ is a solution of (10) and (11) (for the last equality use (7)). Since $1<a<d$, if $\bar{C}^{2}=-1$, then $a=\varphi_{6 j+1}, d=\varphi_{6 j+3}$ and $b=3 d-a=3 \varphi_{6 j+3}-\varphi_{6 j+1}=\varphi_{6 j+5}$ for some $j>0$, by property (7) of Fibonacci numbers. Similarly, if $\bar{C}^{2}=0$, then $\omega$ and $v$ are both either positive or negative which implies $a=\varphi_{6 j+1}^{2}, b=\varphi_{6 j+3}^{2}$ and $d=\omega v=\varphi_{6 j+1} \varphi_{6 j+3}=\varphi_{6 j+2}^{2}+1$.

Case B. If $\gamma= \pm\left(\varphi_{6 j}+\varphi_{6 j-2}-\varphi_{6 j-1} \sqrt{5}\right), j \geq 0$, is a solution of (9) then $v=$ $\pm\left(-\varphi_{6 j-1}\right)$ and $\omega= \pm\left(\varphi_{6 j}+\varphi_{6 j-2}-3 \varphi_{6 j-1}\right) / 2= \pm\left(-\varphi_{6 j-3}\right)$ is a solution of (10) and (11). In the case $\bar{C}^{2}=-1$, one gets $a=\varphi_{6 j-3}, d=\varphi_{6 j-1}$ and $b=3 d-a=3 \varphi_{6 j-1}-\varphi_{6 j-3}=\varphi_{6 j+1}$ with $j>0$. If $\bar{C}^{2}=0$, then $\omega$ and $v$ are both either positive or negative which implies $a=\varphi_{6 j-3}^{2}, b=\varphi_{6 j-1}^{2}$ and $d=\omega v=\varphi_{6 j-1} \varphi_{6 j-3}=\varphi_{6 j-2}^{2}+1$ with $j>0$.

Case C. If $\gamma= \pm\left(\varphi_{6 j+2}+\varphi_{6 j}-\varphi_{6 j+1} \sqrt{5}\right), j \geq 0$, is a solution of (9) then $v=$ $\pm\left(-\varphi_{6 j+1}\right)$ and $\omega= \pm\left(\varphi_{6 j+2}+\varphi_{6 j}-3 \varphi_{6 j+1}\right) / 2= \pm\left(-\varphi_{6 j-1}\right)$ is a solution of (10) and (11). If $\bar{C}^{2}=-1$, then $a=\varphi_{6 j-1}, d=\varphi_{6 j+1}$ and $b=\varphi_{6 j+3}$ with $j>0$. If $\bar{C}^{2}=0$, then $\omega$ and $v$ are both either positive or negative which implies $a=\varphi_{6 j-1}^{2}, b=\varphi_{6 j+1}^{2}$ and $d=\varphi_{6 j-1} \varphi_{6 j+1}=\varphi_{6 j}^{2}+1$ with $j>0$.

Case D. Any solution in this case is included in the previous cases.

Hence, we determined all the possible integer solutions.

5.4. Theorem. Classification for $\bar{C}^{2}=-1$. If $\bar{C}^{2}=-1$ then $(a, b)=\left(\varphi_{j-2}, \varphi_{j+2}\right)$ and $d=\varphi_{j}$, with $j$ odd $\geq 5$. For every such $j$ there exists a unicuspidal rational plane curve of degree with such invariants.

5.5. Theorem. Classification for $\bar{C}^{2}=0$. If $\bar{C}^{2}=0$ then $(a, b)=\left(\varphi_{j-2}^{2}, \varphi_{j}^{2}\right)$ and $d=\varphi_{j-1}^{2}+1$, with $j$ odd $\geq 5$. For every such $j$ there exists a unicuspidal rational plane curve with such invariants. 
We only need to provide the equations of the curves. We will rely on 4, Corollary 11.4. Let $(x, y)$ be a system of affine coordinates in $\mathbb{P}^{2}$ and consider

$$
\begin{gathered}
P_{-1}=y-x^{2}, Q_{-1}=y, P_{0}=\left(y-x^{2}\right)^{2}-2 x y^{2}\left(y-x^{2}\right)+y^{5}, \\
Q_{0}=y-x^{2}, G=x y-x^{3}-y^{3}, Q_{s}=P_{s-1}, P_{s}=\left(G^{\varphi_{2 s+1}}+Q_{s}^{3}\right) / Q_{s-1} .
\end{gathered}
$$

Then $P_{s}$ is a polynomial in $x$ and $y$ of degree $\varphi_{2 s+3}$ and defines a rational unicuspidal curve whose unique singularity $p$ has exactly one characteristic pair of type $(a, b)=\left(\varphi_{2 s+1}, \varphi_{2 s+5}\right)$. The curves $P_{s}=0$ and $Q_{s}=0$ only meet at $p$. The rational pencil with only one base point determined by the rational function $R_{s}=\left(P_{s}\right)^{\varphi_{2 s+1}} /\left(Q_{s}\right)^{\varphi_{2 s+3}}$ has only two special fibres $P_{s}=0$ and $Q_{s}=0$, and the other fibres are rational unicuspidal plane curves of degree $\varphi_{2 s+3} \varphi_{2 s+1}=\varphi_{2 s+2}^{2}+1$. The singularity of a generic fiber has one characteristic pair $(a, b)=\left(\varphi_{2 s+1}^{2}, \varphi_{2 s+3}^{2}\right)$.

\section{REFERENCES}

[1] Fernández de Bobadilla J., Luengo I., Melle-Hernández A., Némethi A.: On rational cuspidal projective plane curves, preprint at arXiv:math.AG/0410611

[2] Dimca, A.: Singularities and Topology of Hypersurfaces, Universitext, Springer-Verlag, New York, 1992.

[3] Fujita, T.: On the topology of non-complete algeraic surfaces, J. Fac. Sci. Univ. Tokyo (Ser1A), 29 (1982), 503-566.

[4] Kashiwara, H.: Fonctions rationelles de type $(0,1)$ sur le plan projectif complexe, Osaka J. Math., 24 (1987), 521-577.

[5] Namba, M.: Geometry of projective algebraic curves. Monographs and Textbooks in Pure and Applied Mathematics, 88 Marcel Dekker, Inc., New York, 1984.

[6] Orevkov, S. Yu.: On rational cuspidal curves, I. Sharp estimate for degree via multiplicities, Math. Ann. 324 (2002), 657-673.

[7] Matsuoka, T. and Sakai, F.: The degree of of rational cuspidal curves, Math. Ann., 285 (1989), 233-247.

[8] Tono, K.: On rational unicuspidal plane curves with logarithmic Kodaira dimension one, preprint.

[9] Tsunoda, Sh.: The complements of projective plane curves, RIMS-Kôkyûroku, 446 (1981), 48-56.

[10] Vajda, S.: Fibonacci and Lucas numbers, and the Golden Section: Theory and Applications, Ellis Horwood Series: Mathematics and its Applications. Ellis Horwood Ltd., Chichester; Halsted Press New York, 1989.

[11] Varchenko, A.N.: On the change of discrete characteristics of critical points of functions under deformations, Uspekhi Mat. Nauk, 38:5 (1985), 126-127.

[12] Varchenko, A.N.: Asymptotics of integrals and Hodge structures. Science rewievs: current problems in mathematics 1983. 22, 130-166; J. Sov. Math. 27 (1984).

[13] Wall, C.T.C.: Singular Points of Plane Curves, London Math. Soc. Student Texts 63, Cambridge University Press, 2004.

[14] Yoshihara, Y.: Rational curves with one cusp (in Japanese), Sugaku, 40 (1988), 269-271.

Department of Mathematics, University of Utrecht, Postbus 80010, 3508TA Utrecht, The NetherLands

Facultad de Matemáticas, Universidad Complutense, Plaza de Ciencias, E-28040, Madrid, SPAIN

Department of Mathematics, Ohio State University, Columbus, OH 43210,USA; And Rényi Institute of Mathematics, Budapest, Hungary.

\footnotetext{
E-mail address: bobadilla@math.uu.nl

E-mail address: iluengo@mat.ucm.es

E-mail address: amelle@mat.ucm.es

E-mail address: nemethi@math.ohio-state.edu; nemethi@renyi.hu
} 